\title{
Lessons Learned From C-141C GPS Enhanced Navigation System (GPSENS) Developmental Flight Test
}

\author{
Capt. John S. Seo \\ 418 Flight Test Squadron \\ Edwards AFB CA 93524 \\ 805-277-7190 ext. 2240 \\ seoj@418flits.edwards.af.mil
}

\author{
Capt. Christopher C. McCann \\ 418 Flight Test Squadron \\ Edwards AFB CA 93524 \\ 805-277-6868 ext. 2985 \\ mccannc@418flits.edwards.af.mil
}

\begin{abstract}
This paper highlights some lessons learned in the developmental testing of the C-141 Global Positioning System Enhanced Navigation System (GPSENS) avionics upgrade. The authors planned and executed the flight tests of the GPSENS systems and learned many valuable lessons about retrofitting modern avionics into older aircraft. They found that commercial off the shelf acquisition strategies seemed much more suitable for hardware upgrades that were not heavily dependent on software. Overcoming limitations in legacy software products proved to be extremely difficult. Also, integrating modern avionics into legacy systems organic to the aircraft was often a hit-or-miss proposition. They learned the value of a high-fidelity engineering simulator and saw the results when the available simulator failed to properly emulate the aircraft in the areas of inertial systems, aeromodels, hardware-in-the-loop and electrical power quality. Finally, in validating a new Kalman filtered navigation solution they developed flight test techniques to improve a system that initially performed very poorly. Their lessons learned should serve as a guide to other engineering professionals embarking on similar development or test efforts.
\end{abstract}

\section{TABLE OF CONTENTS}

\section{INTRODUCTION AND BACKGROUND \\ 2. COMMERCIAL OFF THE SHELF ACQUISITION \\ 3. ORGANIC LEGACY SYSTEMS \\ 4. ENGINEERING SIMULATOR \\ 5. KALMAN FILTERED NAVIGATION SOLUTION 6. LESSONS LEARNED}

\section{INTRODUCTION AND BACKGROUND}

Approximately three years ago, the United States Air Force embarked on a major modification to part of its fleet of Lockheed C-141B Starlifter transport aircraft. Though the vast majority of the fleet was being retired and replaced by the Boeing C-17A Globemaster III, approximately 63 C-141B aircraft were selected to undergo two sequential systems upgrades, the All-Weather Flight Control System (AWFCS) modification and the Global Positioning System Enhanced Navigation System (GPSENS) upgrade. The main purposes of these two modifications were, respectively, to improve the reliability and maintainability of the flight instruments and autopilot and to add Congressionally-mandated GPS navigation capability. This paper will describe lessons learned during developmental flight testing of the GPSENS avionics upgrade.
Genesis of the C-141-The Lockheed C-141B Starlifter, shown in Figure 1, is a subsonic, long-range strategic transport aircraft powered by four Pratt \& Whitney TF-33 turbofan engines. It first flew on 17 December 1963 and has been in operational service with the Air Force since 1965 . In all, 285 aircraft were built. In the early 1980's, modifications were made to most of the fleet; converting them to the $\mathrm{C}-141 \mathrm{~B}$ model. The fuselage was lengthened more than 22 feet and aerial refueling capability was added. Other modifications made up to the present include the installation of two inertial navigation units (INUs) and a Fuel Saving Advisory System (FSAS) computer, essentially an early version of a flight management system (FMS). The mission of C-141B includes long-range strategic airlift, tactical airlift, combat airdrop, and special operations.

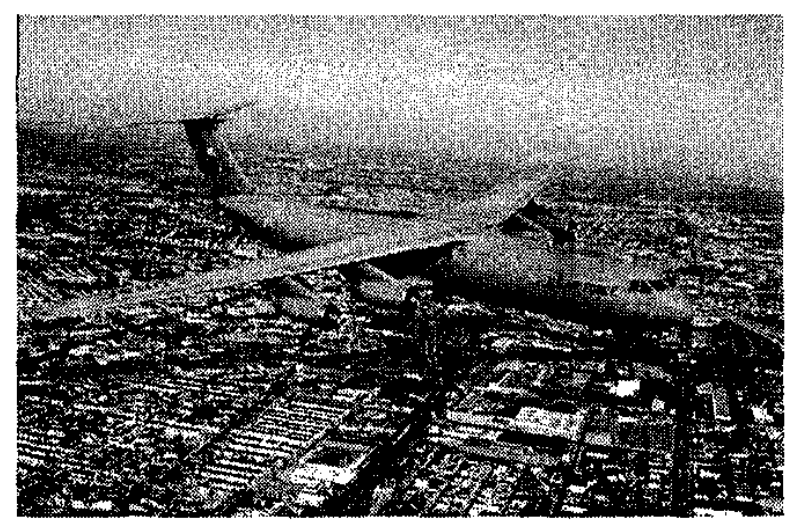

Figure 1 USAF C-141C Starlifter

AWFCS-In the early 1990's the Air Force began to experience problems in maintaining the electromechanical flight instruments the pilots use as well as the autopilot. The age of the systems, the lack of spare parts, and the huge surge in flying brought on by the Gulf War combined to critically impact aircraft mission readiness. To improve reliability and maintainability, the Air Force upgraded the cockpit under the AWFCS contract. The revamped flight station now included four 6 inch by 8 inch active-matrix liquid crystal displays (AMLCD) in place of the older flight instruments, two new digital autopilots, a new digital ground collision avoidance system as well as a new self-contained AMLCD multifunction standby instrument (MFSI), a 
combined attitude indicator, airspeed indicator and altimeter. Figure 2 is the cockpit display of the GPSENS test aircraft.

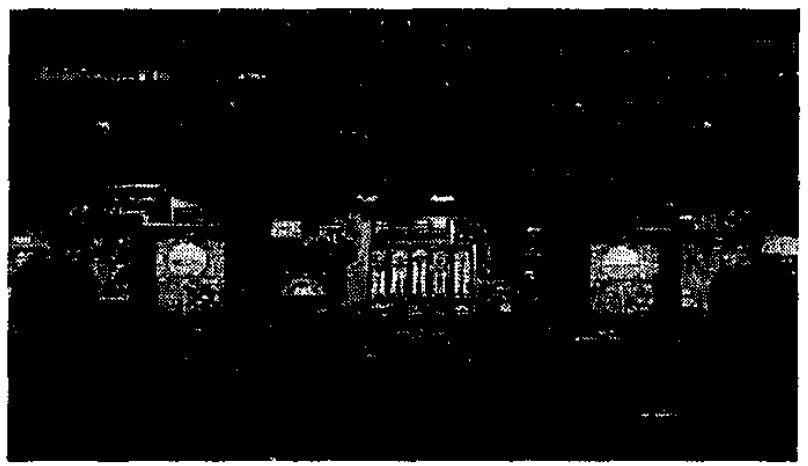

Figure 2 C-141C Glass Cockpit

GPSENS-As a result of the investigation into the 1995 crash of an Air Force T-43 aircraft in Croatia, Congress mandated in its GPS-2000 directive that all military transport-type aircraft be upgraded GPS navigation capability by the year 2000 . To meet that mandate, as well as to update the flight management capability of the C-141B and facilitate future enhancements, the GPSENS modification was developed. Included in this upgrade were dual embedded GPS receivers (EGRs) and the installation of two COTS FMS computers. The combined AWFCS/GPSENS upgrades made the modified aircraft sufficiently different from its original pedigree to warrant a new type, and the C-141C was born.

The $418^{\text {th }}$ Flight Test Squadron at Edwards Air Force Base, California, was tasked with performing the developmental and qualification flight testing of the C-141C AWFCS and GPSENS modifications. Developmental testing essentially consists of aiding the contractors in fine-tuning and troubleshooting their design using the real aircraft as a flying laboratory. During qualification testing, the test pilots and flight test engineers thoroughly evaluate the entire system to verify it meets all design specifications, to assess its impact on human factors, and to determine if the new system is operationally suitable for its intended mission.

Developmental testing of the GPSENS modification has been completed and qualification testing is in progress. Along the way, the authors learned several valuable lessons about flight testing modern avionics systems integrated into a very mature (read: old) airframe. A lot can be learned by highlighting some key areas of the lessons learned, not just by other flight testers, but by any engineer or manager who gets involved with a similar program. A suitable subtitle for this paper would be: How to teach an old dog new tricks... and not get too badly bitten in the process.

\section{COMMERCIAL OFF THE SHELF ACQUISITION}

In the days of free-flowing defense dollars nearly every major system the military purchased was specifically designed for the military. Those days are long gone, and affordability is now a key factor in the acquisition of defense systems. One way to potentially save a lot of money up front in development costs and possibly over the life of a program, is to purchase commercially-produced equipment and use it in a military capacity directly or with minor modification. This concept is called COTS. In its most utopian form, the Air Force could purchase a mass-produced commercial aircraft, paint it NATO gray and press it into military service with no other modifications. In theory, acquisitions using COTS result in shorter development times, easier sustainability, and lower lifecycle costs because the military would be getting the best the competitive commercial aerospace industry has to offer at good prices.

COTS in GPSENS-COTS was a major driver in the GPSENS program, since for years thousands of civilian airliners have been equipped with GPS navigation systems and FMS computers. It offered the potential for quickly modifying the $\mathrm{C}-141 \mathrm{C}$ with proven, affordable, available hardware. All that was required for the software that drove the boxes was to "missionize" or tailor the code to support the missions the $\mathrm{C}-141 \mathrm{C}$ performed in operational service.

A COTS Success-In the AWFCS modification a selfcontained Multi-function Standby Instrument (MFSI) was installed in the instrument panel. This backup instrument was a 3-ATI-sized LCD unit with its own gyroscope, air data computer and display driver. It received raw Pitotstatic inputs and electrical power from the aircraft. Minor software modifications were made to the attitude, airspeed, and altimeter displays to make them look like the new electronic primary flight display the pilots normally used. The MFSI proved to be extremely reliable and represented a major improvement in the aircraft's backup systems. The altimeter display even included the metric equivalents of the normal altitude and barometric altimeter setting units. The only integration the MFSI had with the aircraft's existing systems were the Pitot-static inputs, electrical power and a reference voltage for display dimming.

Software Legacies-The FMS multifunction control and display units (MFCDU's) were commercially developed and had been in use on the Boeing 777 aircraft for several years. The hardware, though it was not designed specifically for the $\mathrm{C}-141 \mathrm{C}$, worked well and supported the aircraft's mission adequately. The FMS legacy software was another story entirely. The software that drove the FMS was a combination of mostly Boeing 777 code developed for commercial airlines and some specific military mission functionality designed for Belgian Air Force C-130 Hercules aircraft. This marriage proved to be a troublesome union.

The COTS Tradeoff-The use of COTS software necessarily reduced or eliminated the ability of the end users to make input early in the design phase of the program. This ability to specify operating details is supposed to be outweighed by the savings in time and money during development. Much of the functionality that would have been designed from the bottom up in a developmental program was assumed to exist or could easily be added by "just making a software 
change." Very few avionics systems contain the detailed system operating characteristics early on that a mature system will eventually have. This was clearly the case in the development of the GPSENS FMS software.

The 777 Legacy-The typical commercial airliner departs from a civilian airport, climbs to its cruise altitude, descends, and then lands at its intended destination. This standard flight profile does not even begin to resemble the typical mission profile of a C-141 aircraft. A Starlifter mission could include a formation takeoff, high-altitude formation cruise flight, multiple climbs and descents for aerial refueling, low-level navigation ingressing to a tactical drop zone for an airdrop, a climb back to cruise altitude and finally a descent to the final destination.

During flight test, the FMS flight profile model was found to be almost incapable of performing the $\mathrm{C}-141 \mathrm{C}$ mission. The software required the aircraft to follow a very rigid profile: climb, cruise, followed by a descent. It was possible to transition the vertical navigation (VNAV) system from descent back to climb, but not vice versa. Several other idiosyncrasies of the VNAV system reflected the 777 way of doing business almost at the exclusion of the C-141C mission. It became apparent that in order to properly missionize COTS software for a different end user, engineers had to first understand what that mission would entail and how it would be flown. The desire to initially accept COTS software without proper regard to the specific mission it was intended to support resulted in major modifications being recommended late in developmental testing. Schedule and cost pressures were tremendous at that point, not exactly an ideal environment for a major software rewrite.

The C-130 Legacy-The Belgian C-130 program's contribution to the GPSENS FMS software was mainly in the form of airdrop and landing zone functions and takeoff and landing data (TOLD) computations. While the airdrop and landing zone operations were very similar between the C-130 and C-141C, the TOLD was not. Digitizing the C-141C performance data charts was not horribly difficult. However, the required performance calculations differed greatly between a four-engine turboprop tactical airlifter and a turbofan-powered, long-range strategic transport. The attempt to force the square-pegged $\mathrm{C}-130$ TOLD into the round-holed C-141C model resulted in a minimally capable system that is still being modified. Again, the desire not to change COTS software appeared to override the requirement to perform the specific mission. Maximum involvement of the end user early in the process may have alleviated some of these problems.

Performance Model Differences-The basic performance models of the 777 and the C-130 are vastly different, while the $\mathrm{C}-141 \mathrm{C}$ would appear to be somewhere between the two. Marrying the 777 and C-130 performance models probably seemed like a good approximation of the C-141C. Our testing essentially verified that assumption, but some key differences surfaced.

U.S. Government work not protected by U.S. copyright.
Autothrottles-Autopilot-coupled descents in the Boeing 777 were typically performed with the autothrottles engaged. This was not possible in the $\mathrm{C}-141 \mathrm{C}$ because the aircraft required a minimum thrust setting on at least one engine on each side of the aircraft to maintain cabin pressurization. The inability to use the autothrottles during descent at first seemed to be a minor inconvenience. As testing progressed into vertical path descents, the error of our ways became evident. Without the FMS controlling the autothrottles in a path descent, the pilot had to attempt to modulate thrust as the aircraft varied pitch to follow the descent profile. Without any type of thrust director systems it was very difficult to maintain the desired descent speed to within even 10 knots, and large changes in airspeed, as in slowing to 250 knots at 10,000 feet MSL, were nearly impossible. More than 10 hours of flight test were devoted to trying to solve this problem.

Drag Model-Anyone who's ever seen a C-130 up close can say the Hercules is not the sleekest craft plying the skies. The four large props that beat the air into submission are drag factories, as are its blunt features, outcroppings of antennae and wires, and its main landing gear blisters. While the C-141C won't win any awards for slipperiness, it's no Herc. Computations in the FMS that relied on accurate drag estimations, such as slowing the aircraft down to enter holding, configure for an airdrop, or meet a flight plan speed constraint, were way off. Since an accurate aeromodel of the C-141 was not handy, the test team had to go the road of empirical flight test, also known as 'trial and error.' The system was eventually tweaked to provide tolerable performance but at the cost of many flight hours that were never anticipated.

One Speed Fits All-Another regime in which our testing showed a shortcoming of COTS software was in the area of default speed selections. Going back to the typical airline cruise profile, once the aircraft reaches altitude it settles into a specific cruise Mach number, usually chosen to provide maximum fuel economy with a reasonable ground speed. The manifestation of this legacy profile in the C-141C was the selection of a 'default' FMS cruise speed of 0.74 indicated Mach number (IMN). In fact, $0.74 \mathrm{IMN}$ is the typical cruise speed of the C-141C at normal cruise altitudes above 29,000 feet Mean Sea Level (MSL). The test pilots were quite surprised when they put the aircraft into cruise mode at 8,000 feet MSL and the aircraft immediately accelerated to 350 knots calibrated airspeed (KCAS), the aircraft's limiting airspeed. While the average civilian airliner would almost never 'cruise' at 8,000 feet MSL, C$141 \mathrm{~s}$ quite often fly at such altitudes during training missions. A detailed examination of the mission impacts of every function in the COTS software may have caught this problem earlier.

In theory COTS is a good idea, and there were successes in the GPSENS program, particularly with regard to hardware. But when it came to software, our experience was that COTS could actually be an acronym for 'count out the software.' The overriding desire to save time and money through COTS was diametrically opposed to the concept of 
custom-tailoring the system to the intended mission. The impact of incompatible legacy systems, and a failure to fully missionize the software, resulted in huge expenditures of extra flight time and test planning.

\section{ORGANIC LEGACY SYSTEMS}

Any program that involves integrating new avionics into an older aircraft will have to face the decision of what original, organic aircraft systems will stay and what will be replaced or upgraded. It would seem obvious that the more organic equipment that is retained the cheaper and easier the modification would be, as existing spare parts, technical data, maintenance procedures and other existing support could still be used. The GPSENS modification showed that this was a blessing in some cases and curse in others.

The Standardized Central Air Data Computer Success-The standardized central air data computers (SCADCs) provide barometric altitude, airspeed and vertical velocity data to the flight displays. The original SCADCs were retained in the GPSENS modification since they were fairly reliable and maintainable. By not replacing them the test team avoided having to completely recalibrate and recertify the aircraft's entire Pitot-static system, a very involved, labor-intensive set of flight tests. The SCADCs also provided fairly clean, reliable digital outputs to the various new components added in the GPSENS and AWFCS modifications.

Inertial navigation units-We expected similar success in retaining the original Carousel IV gyroscopic inertial navigation units (INUs). The INUs had been in service on the C-141B for well over a decade and were known quantities. They were reliable and had proven to be good sources of heading, attitude, airspeed, and position data for the flight displays and other systems. The first hint of trouble came when we did our initial analysis of the accuracy of the Kalman navigation solution (KNS), a computer-filtered, GPS-aided inertial position reference. The KNS was supposed to be the 'Holy Grail' of navigation solutions, combining the best aspects of the GPS and INU solutions. To our dismay, the KNS was orders of magnitude worse than the raw GPS position.

Contractor engineers discovered that the position and velocity data fed into the Kalman filter from the INU's was not time-synchronized properly, resulting in large errors and data rejection in the Kalman algorithm. To make matters worse, the contractors were unable to locate any of the original INU technical specifications, and the original manufacturer was unable to help. This problem was eventually overcome, but not until several dedicated test sorties were flown and many hours of data were collected. The legacy INUs proved to be a liability when integrated with modern avionics. A detailed discussion of how we solved the KNS problem will be presented later in this paper.

Aircraft Electrical System-Some legacy equipment issues are impossible to avoid, as was the case with the aircraft's electrical power system. It was designed in the early 1960's and, as a fundamental, embedded part of the aircraft, was changed little over the years. The quality of the electrical power was more than adequate for the analog and electromechanical subsystems that made up the majority of the aircraft. Modern digital avionics, however, proved to be particularly intolerant of less-than-clean power and sporadic power transients.

Power Transients-We discovered that modern COTS digital avionics designed for new aircraft had trouble accounting for the normal power interrupts of an older aircraft. Multiple software revisions had to be generated to attempt to handle warm and cold system restarts as a result of a power transient. These system reboots manifested themselves as numerous nuisance fault indications as the individual components each ran their own power-on built-intests (BITs). Such nuisance indications were never completely eliminated. Rather, we were able to quantify what was a 'normal' nuisance indication and what was not.

Emergency Generator-The C-141 has a hydraulically powered emergency generator to supply backup AC and DC power in the event that all four engine-powered generators are lost. This 2 kilo volt (kVA) generator was adequate to provide enough power in an emergency situation in the unmodified aircraft, but an electrical loads analysis conducted prior to the modifications indicated it would be overloaded with even a minimum number of the new systems powered. The workaround to this problem was a change in operating procedures that allowed the emergency generator to power only some electronic flight displays while de-powering the remainder. The original aircraft flight instruments included electromechanical gyroscopic attitude director indicators (ADI) for the pilot and copilot. Even in the event of complete electrical failure, the gyro in the ADI would slowly spin down and provide reliable attitude information for 9 to 10 minutes. Fortunately, the addition of the new MFSI backup instrument, and its direct wiring to the aircraft battery, alleviated most of that problem.

Similar to the COTS issue, engineers have to consider the impact of organic aircraft legacy systems. Exceptional creativity and ingenuity were required to overcome the problems we experienced.

\section{ENGINEERING SIMULATOR}

A high-fidelity engineering simulator is worth its weight in gold when working on developmental aircraft systems. In preparation for designing and testing the AWFCS and GPSENS systems, the contractor built a hardware-in-theloop engineering simulator. This fixed-base simulator allowed the contractor engineers to integrate nearly all of the new system components and stimulate them with test inputs to evaluate system functionality. Since the simulator included many pieces of actual hardware, such as the display units and various system processors, it essentially evolved alongside the system it was intended to simulate. This occasionally made it difficult to tell whether an unexpected result was a problem with the system or the simulator. Further, we discovered that accurately simulating a complex 
inertial system with a low-fidelity, fixed-base simulator was not always very helpful in troubleshooting.

Modeling an Inertial System-While the simulator did make use of many of the actual system components, the nature of the inertial system being emulated did not allow true high-fidelity simulation. The inertial navigation system inputs, which would come from the INUs on the aircraft, had to be modeled, since the simulator was bolted to the floor of the contractor's laboratory. An accurate INU model proved very difficult to obtain since even the original equipment manufacturer was unable to produce one. Approximations and engineering judgments had to be made when modeling the INU inputs for the simulator.

While the INU model was not perfect in some ways, it was too perfect in others. As mentioned previously, a problem was discovered with the interface between the INUs and the Kalman navigation algorithm that stemmed from the lack of good time synchronization between the INU position and velocity outputs. The INU model used in the engineering simulator did not have this shortcoming, so the KNS solution in the simulator was always top-notch. The engineers also found it to be difficult to accurately model the time synchronization problem to allow them to troubleshoot it on the engineering simulator. One of the many results of the limitations of the low-fidelity simulator was a large increase in developmental flight test hours devoted to solving the INU-KNS interface dilemma.

Hardware-in-the-Loop-One of the tenets of conducting simulations with actual flight hardware is actually including all of the important parts of the system in the simulator. Simulation engineers have to fully analyze and weigh any shortcuts they take in trying to build a cost-effective, useful simulator. Sometimes this analysis fails to catch the small details that will later drive you to distraction. The GPSENS simulator was a good example of this.

Dual Boxes-The design of the navigation processing segment of the system includes two separate, identical navigation processors (NPs), Each NP maintains its own navigation solution, performance predictions, vertical guidance calculations and other system data. The two NPs continuously monitor each other for validity and compare their respective solutions. This necessitates a lot of communication and bus traffic between the two line replaceable units (LRUs), or boxes.

Since the GPSENS engineering simulator was actually evolving as the boxes were coming off the production line, the availability of flight-qualified hardware was poor. For this reason, and to simplify the NP interface to the simulator, the engineers initially elected to only include one NP LRU in the engineering simulator and to model the other one. During flight test, a host of dual-box communication problems were encountered that often resulted in the two NPs being completely out of synch with each other. The contractor engineers were initially unable to model this behavior in the simulator because it did not truly include the dual-box system.
The decision was made to upgrade the engineering simulator to the actual dual-box configuration. Shortly thereafter, the sources of the NP communication difficulties were uncovered and corrected. Further, the dual-box setup actually allowed the engineers to fully regression test new software loads. This reduced the likelihood of problems remaining undiscovered on the simulator only to pop up on the aircraft. Literally dozens of hours of flight test were saved by performing full-scale regression testing on the improved simulator.

Clean Power-Let's go back for a moment to the difficulty of integrating modern digital avionics into an aircraft with less-than-perfect electrical characteristics. The problem on the aircraft was that the quality of the power was low compared to what the avionics were originally designed to handle. The opposite problem was true in the engineering simulator. The power fed into the simulator and all its LRUs was controlled, conditioned, and uninterrupted - a less than faithful model of the real aircraft. Attempts were made to better simulate the 'colorful' properties of the C-141's electrical power, but the engineers were not nearly as effective at degrading and corrupting the power supply as the venerable Starlifter.

This inability to truly model the electrical properties of the actual aircraft required us to perform all the power quality tolerance testing on the jet. Even attempting to use another $\mathrm{C}-141 \mathrm{C}$ at the contractor's facility proved vexing, as we learned that individual aircraft displayed their own distinct electrical characteristics. Solutions to electrical power problems proved to be very elusive and particularly difficult to solve or simulate across a fleet of 63 aircraft that averaged 33 years and over 20,000 flight hours each. Our approach to testing these solutions resembled the brute force method - ground test, fly, fix, ground test, fly, fix ..., an inelegant method, but our only option given the inability to accurately simulate the problem.

My Kingdom for an Aeromodel-The era that produced the Starlifter was the era of the sliderule. High-speed digital computers and computational fluid dynamics were just a gleam in the aerodynamicist's eye. The concept of aeromodeling was just beginning to evolve. Aeromodeling is defined as empirically deriving a mathematical model of an aircraft's aerodynamic and dynamic characteristics and validating that model through flight test. Without question, aeromodeling is expensive and time consuming and as a consequence is avoided whenever possible. Why would a simple autopilot and FMS require an accurate aeromodel?

A true aeromodel of the C-141 did not exist when the AWFCS and GPSENS modifications were begun, nor does one exist today. In its place, the contractor engineers used a 'generic large aircraft aeromodel' in developing the flight control laws and related software for the aircraft. Being a purely military aircraft there was not a comparable civilian equivalent to the high-swept-wing, T-tailed, turbofanpowered, long-range C-141C. Any 'generic' aeromodel was guaranteed to have at least a few shortcomings. 
Flying Qualities-The combination of a low-fidelity, fixedbase simulator and an inaccurate aeromodel masked most of the deficiencies in actual autopilot-coupled flying qualities. The roll-rate model did not faithfully describe the C-141's lumbering, tortoise-fast roll qualities. Simulator testing of the autopilot's lateral modes did not reveal the oscillatory lateral tracking of the FMS navigation solution. Performing engineering evaluations of flight control law gain changes in the simulator was of little value and was eventually abandoned.

Gain Sweeps-Our solution to pinning down the proper gains for the autopilot was a series of gain sweeps. Problematic maneuvers, such as intercepting an FMS course between two flight plan waypoints when only slightly off course, were repeated multiple times with various control law gain changes. The contractor engineer was able to directly change the gains in-flight for preselected parameters within upper and lower bounds that were set prior to flight. The control law specialist was usually able to secure acceptable performance using the available gains within the available ranges. However, every time he was not able to do so required a software change to increase the number and or range of the gains that could be modified. And a software change translated directly into another flight test sortie.

Similar gain change flight tests were conducted to improve autothrottle performance, especially in turbulence. The vertical guidance control laws were especially nettlesome due to large disparities between the aeromodel's pitch characteristics and the aircraft's true flying qualities in the pitch axis. On several occasions at similar flight conditions, the aircraft demonstrated a lightly damped, high-frequency pitch oscillation when the autopilot's altitude hold mode was engaged. On other occasions the pitch oscillations were very slightly divergent.

Attempts to purposely drive the aircraft into this situation in flight were not very successful. Neither of these pitch modes was evident in the simulator, nor could they be reproduced in the simulator even after thoroughly analyzing the gigabytes of flight test data that were recorded during the sorties where the oscillations were encountered. Unable to consistently reproduce the problem in aircraft, and completely unable to recreate it in the simulator, we opted to forego trying to fix the problem with gain sweeps.

\section{KALMAN FILTERED NAVIGATION SOLUTION (KNS)}

The GPSENS modification included the addition of a KNS that could be used for aircraft navigation. The Kalman filter algorithms were developed and successfully applied in the field of navigation since the genesis of the Starlifter. Kalman filter algorithms provide means to consider random processes such as aircraft maneuvering by modeling them in vector form (as in equations of motion) and using recursive processing (taking advantage of high speed digital computing) to minimize noisy measurement data through error estimations. [1]
Theoretically, KNS position and velocity should be at least as accurate as the GPS and INS inputs. High degrees of navigational accuracy are critical to several of the C-141C's missions, especially airdrop, landing zone operations, and enroute navigation in minimum navigation performance specification (MNPS) airspace such as the North Atlantic oceanic region.

KNS Accuracy-Initial KNS navigation accuracy results were surprisingly disappointing. Compared to the certified differential-GPS (D-GPS) truth source carried on board the aircraft, the KNS was more than 150 meters (2-Dimensional Root Mean Square) off. The GPS position displayed accuracy on the order of 5 to 10 meters (2-D RMS). Clearly there was a problem with the KNS.

Latency Issues-The KNS employed in GPSENS was an open-loop, feed-forward Kalman filter system. A block diagram of this system is shown in Figure 3.

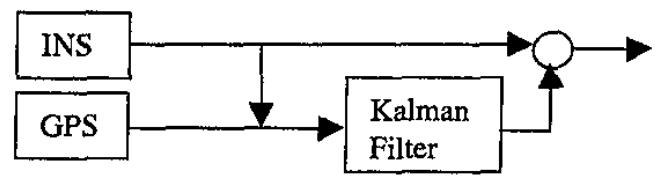

Figure 3 Open-loop Feed-Forward Kalman Filter

This particular Kalman filter design assumed that INS and GPS position and velocity values were measured at the same instance in time, i.e., had the same time of validity. Close examination of the flight test data revealed that the INS data had a different time of validity from the GPS output. In fact, unlike the GPS, we found the venerable Carousel-IV INUs did not provide any time tag with its position and velocity data. A search for technical data on the Carousel-IV did not reveal any information describing INU position and velocity time tags. It became apparent that the gross difference between KNS and GPS position accuracy could be attributed to some undocumented and unquantified position and velocity data latency. Lacking any detailed technical specifications describing this latency led us to looking for empirical methods to quantify and correct this error.

Latency Corrections-The difference between INS and GPS time of validity was termed 'position $\Delta 2$ ' to reflect the resulting difference between the INS and GPS positions. This 'position $\Delta 2$ ' value was effectively an adjustment to the INS data time of validity to properly synchronize INS and GPS inputs into the Kalman filter. A basic description of how an inertial navigation system calculates position helps to explain the flight test technique (FTT) that was used to empirically derive the best value for 'position $\Delta 2$ '.

Inertial Navigation Basics - Inertial navigation systems employ dead reckoning (DR) to calculate position from velocity measurements. The position of the aircraft is initially loaded in to the INS and the position is then continuously recomputed by integrating measured ground speed. In a simple system, neglecting the effects of wind, a DR navigation system can calculate the easterly and 
northerly components of position over the surface of the Earth using ground speed $\left(\mathrm{V}_{\mathrm{GS}}\right)$ and true heading $\left(\mathrm{W}_{\mathrm{T}}\right)$ by Equations (1) and (2) below.[2]

$$
\begin{aligned}
& V_{\text {EAST }}=V_{G S} \operatorname{Sin} W_{T} ; x=\int_{t 0}^{t 1} V_{E A S T} d t \\
& V_{\text {NORTH }}=V_{G S} \operatorname{Cos} W_{T} ; y=\int{ }_{t 0}^{t} V_{\text {NORTH }} d t
\end{aligned}
$$

Inertial navigation systems resolve velocity into east and north components using gyroscopes and accelerometers. These east and north velocity components are then integrated over time to find position and this position is, inturn, supplied to the Kalman navigation algorithm.

Optimizing 'Position $\Delta 2$ '- To find an optimum value for 'position $\Delta 2$ ' we attempted to minimize error and noise in the INS position data by effectively eliminating the east or north velocity terms independently. This was accomplished by maintaining a true cardinal heading while recording position data. For example, the aircraft was flown on a constant $360^{\circ}$ true heading (due north), effectively driving $V_{\text {EAST }}$ to zero. This allowed the contractor engineer to tweak the 'position $\Delta 2$ ' value in real time to match up INS and GPS northern position values. This same process was used traveling due east, due west, and due south. This flight test technique was appropriately dubbed 'boxes in the sky.' These four values for 'position $\Delta 2$ ' were then numerically averaged and this final value of 'position $\Delta 2$ ' was then, evaluated during a few more iterations of boxes in the sky for post flight comparison to the D-GPS truth source.

'Velocity $\Delta 2$ '-The accuracy improvement was impressive. However, the KNS navigation solution was still less accurate ( $>20$ meters 2-D RMS) than the GPS. Further, the Kalman algorithm was still occasionally rejecting velocity inputs during dynamic maneuvering. Detailed analysis of flight test data showed the Kalman algorithm was failing its three-sigma $(3-\sigma)$ velocity reasonableness test. When the Kalman filter compared INS and GPS velocity values and detected that they were outside the 3- $\sigma$ tolerance, it rejected the GPS velocity inputs. Further analysis revealed INS velocity data latency was not the same as position latency. By introducing a separate velocity latency correction term, 'velocity $\Delta 2$ ', the velocity data could be synchronized in time with the position data prior to feeding it to the Kalman filter.

Optimizing 'Velocity $\Delta 2$ ' - In order to determine the optimal value of velocity $\Delta 2$, another FTT was employed, this one affectionately called 'circles in the sky.' It required the aircraft to perform several continuous constant-speed, maximum rate, $360^{\circ}$ level turns to the left and to the right. The purpose of the turns was to introduce a maximum rate of change of aircraft true heading while maintaining approximately constant ground speed. Referring again to Equations (1) and (2), this FTT resulted in a rapid and continuous changes in $\mathrm{V}_{\mathrm{EAST}}$ and $\mathrm{V}_{\mathrm{NORTH}}$. These velocity component changes were considered the worst case scenario that would be fed into the Kalman algorithm and would stress the $3-\sigma$ velocity reasonableness test.
The goal was to find a 'velocity $\Delta 2$ ' value that brought INS velocity data to within 3- $\sigma$ accuracy of the GPS velocity in this worst cased dynamic maneuver. Once a value of 'velocity $\Delta 2$ ' was found that eliminated Kalman filter velocity data rejection, that value was further optimized to enhance overall KNS position accuracy. A change in the 'velocity $\Delta 2$ ' value of 0.01 second would improve the position accuracy by the product of $\mathrm{V}_{\mathrm{GS}}$ and this change in the velocity $\Delta 2$. For example a 0.01 -second change in velocity $\Delta 2$ with a ground speed of 220 meters per second $(\mathrm{m} / \mathrm{s})$ (430 knots) would improve position accuracy by 2.2 meters. The final position and 'velocity $\Delta 2$ values were then used in a complimentary filter between the INS and the Kalman filter as shown in Figure 4.

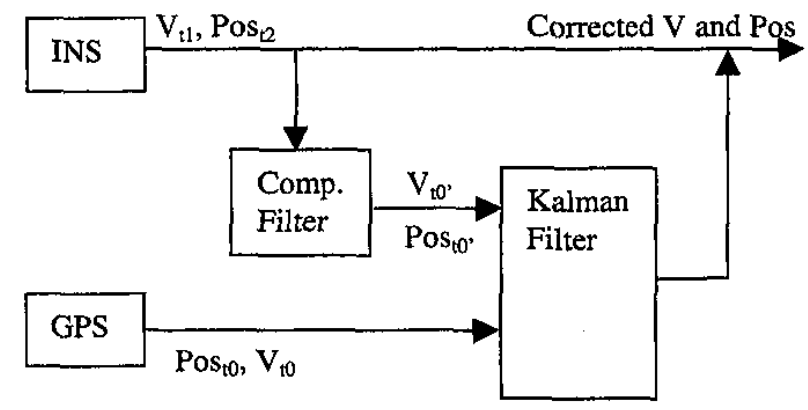

Figure 4 Complimentary Filter for KNS

The improvement in accuracy gained by adding the 'velocity $\Delta 2$ ' value was not as dramatic as the gains from adding the 'position $\Delta 2$ ', but the KNS position was now on the same order of magnitude as the GPS solution. Table 1 illustrates the improvements in position accuracy due to the addition of the $\Delta 2$ values.

Table 1. Navigation Accuracy for KNS

\begin{tabular}{|c|c|c|c|}
\hline $\begin{array}{c}\text { Accuracy } \\
\text { in meters } \\
\text { (2-D RMS) }\end{array}$ & Baseline & $\begin{array}{c}\text { Position } \\
\Delta 2\end{array}$ & $\begin{array}{c}\text { Position } \Delta 2 \\
+ \\
\text { Velocity } \Delta 2\end{array}$ \\
\hline KNS & $>150 \mathrm{~m}$ & $>20 \mathrm{~m}$ & $10-15 \mathrm{~m}$ \\
\hline GPS & $5-10 \mathrm{~m}$ & -- & - \\
\hline
\end{tabular}

Sufficient KNS Accuracy-While the flight test data showed the resulting 2-D RMS accuracy of the KNS position to be on average less than the accuracy of the precision-coded GPS position, it should be noted that the accuracy of the GPS position is very dependent on the number of GPS satellites in view and their geometry relative to the aircraft. In some geographic locations, the GPS solution will have a worse figure of merit and higher estimated horizontal error than the KNS solution. In these real-world situations, the KNS solution will most likely be more accurate then the GPS and would be used for aircraft navigation.

It was for these reasons that further optimization of position and 'velocity $\Delta 2$ ' values was not accomplished. The added expense of performing more flight tests to improve KNS position accuracy below 10 meters was deemed unnecessary; since the level of accuracy achieved was sufficient for real-world operations. Our experience in 
solving the KNS accuracy problem once again validated the usefulness of flight test in the absence of analytical methods and key technical data.

\section{LESSONS LEARNED}

The economic realities of developing completely new aircraft dictate that retrofitting modern technology into older aircraft is and will be common practice. These avionics upgrades are driven by the need to take advantage of new technologies to make operations safer and more efficient. New operating requirements, such as Reduced Vertical Separation Minima (RVSM) and General Air Traffic Management (GATM), will require older aircraft to be retrofitted with new equipment simply to operate in the same airspace as more modern aircraft. While the GPSENS modification to the $\mathrm{C}-141 \mathrm{C}$ was primarily driven by reliability and maintainability concerns and Congressionallymandated system enhancements, it also represented an opportunity to modernize much of the Starlifter's avionics with an eye for future growth. Our experiences in performing GPSENS developmental flight test proved that you can, in fact, teach an old dog new tricks, but you have to be well prepared for the lessons.

The Lure of COTS-Commercial, off-the-shelf acquisition seemed to work well for hardware but was problematic when it came to the software inside the box. The desire to save time and money in development through COTS cannot be allowed to grossly compromise the end-user's intended mission, especially when that mission has been executed for over 30 years. Legacy systems, particularly software, have to be fully analyzed with the new user in mind. Failure to do so will undoubtedly lengthen and complicate any development flight test efforts.

Mixing Old and New-From an engineering perspective one can never underestimate the importance of fully understanding how organic systems will integrate with systems that are generations newer. System developers have to carefully weigh perceived cost and time savings of not upgrading older components against some very thorny integration issues. Be prepared to develop workarounds and new interfaces on the fly and always expect flight testing to take longer than expected because of those fixes. Certain inherent limitations, such as an old, dirty electrical power system, are unavoidable and will have to be dealt with headon.

Simulation, Simulation, Simulation-Nearly everyone seems to understand the importance of high-fidelity simulation, but putting it into practice can be tricky. The value of a mature high-fidelity engineering simulator that accurately models the system in development cannot be overstated. All relevant aspects of the system must be accurately simulated, through true hardware-in-the-loop design that includes all the hardware, accurate aeromodels and inertial inputs, and an accurately good and bad electrical power simulation. Recognition of the inherent limitations in using a fixed-base engineering simulator to develop inertial systems is a must. The upfront investment in time, money and equipment will invariably pay off in any complex, long-term development effort. The ultimate degree-of-freedom simulator, the actual aircraft, can be used in lieu of a good engineering simulator, but the monetary and scheduling costs will be high. Our experience showed an order of magnitude increase in the number of test flights required over what was initially proposed by the contractor, mostly due to the inability to accurately simulate the aircraft in the engineering lab.

Kalman Navigation Solution-The addition of a KNS to the C-141C was supposed to represent a quantum leap in available navigation accuracy over the purely inertial Carousel-IV INUs in the C-141B. In the end this was the case; but it was a long and winding road to get there. In the absence of detailed technical specifications for the CarouselIV, assumptions were made about the INS inputs into the Kalman filter; assumptions that proved to be incorrect and difficult to overcome. Through empirical flight test methods and a bit of creativity on the part of the combined government-contractor test team, flight test techniques were applied that led to the development of a complimentary filter for the original Kalman filter that minimized the time latency problems of the INU position and velocity outputs. These problems would most likely not have been solved without the disciplined application of flight test methodology.

Taken together, these lessons learned form the beginnings of a checklist of issues that should be evaluated before embarking on any moderate- or large-sized avionics upgrade program, especially one involving fairly old aircraft. Engineers and managers involved in all aspects of the design, development, production, and testing of such systems have to grasp the relative merits and impacts of seemingly minor decisions. The old dog can still bite, and it's probably had a lot of practice.

\section{References}

[1] Brown and Hwang, Introduction to Random Signals and Applied Kalman Filtering, Wiley, 1997.

[2] Kayton and Fried, Avionics Navigation Systems, $2^{\text {nd }}$ Ed., Wiley, 1997.

Captain John S. Seo is an instructor flight test engineer assigned to the $418^{\text {th }}$ Flight Test Squadron at Edwards Air Force Base, California. He is the Chief C.141 Operations Engineer for all $\mathrm{C}-141$ test programs. $\mathrm{He}$ graduated from the University of Michigan in 1990 with a BS and earned his $M S$ in aerospace

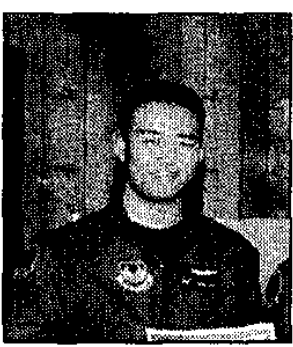
engineering in 1991. He is a graduate of the USAF Test Pilot School, Class $96 B$. 
Captain Christopher C. McCann is a C-17 and C-141 instructor test pilot assigned to the $418^{\text {th }}$ Flight Test Squadron at Edwards Air Force Base, California. He is the lead test pilot for the C-141 AWFCS and GPSENS programs. He served as an operational $C-141 B$ pilot for $41 / 2$ years at Charleston $A F B$, South Carolina. He is a 1988 graduate of the US Air Force Academy and has a BS degree in astronautical engineering. He graduated in

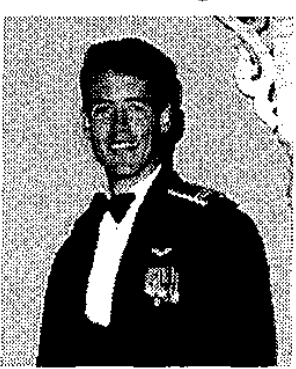

December 1995 from the USAF Test Pilot School in Class 95A. He is currently working toward an MS in aerospace engineering through the University of Alabama. 